DOI: http://dx.doi.org/10.18524/1810-4215.2017.30.114274

\title{
MOLYBDENUM AND RUTHENIUM ABUNDANCES IN COOL STARS OF THE GALACTIC DISC
}

\author{
T.I.Gorbaneva, T.V.Mishenina \\ Astronomical Observatory, Odessa National University, Odessa, Ukraine \\ clumpstars@ukr.net,tmishenina@ukr.net
}

\begin{abstract}
We revise the molybdenum and ruthenium abundances in FGK stars with metallicities ranging from $-1.0<[\mathrm{Fe} / \mathrm{H}]<+0.3$. The observed stars belong to the substructures of the Galaxy. The observations were conducted using the $1.93 \mathrm{~m}$ telescope at Observatoire de Haute-Provence (OHP, France) equipped with the echelle type spectrographs ELODIE and SOPHIE. The results are based on analyses of spectra that have a typical $\mathrm{S} / \mathrm{N} \sim$ 100-300 and a resolution of 42000 . These estimates were obtained using synthetic spectra computed with LTE model atmosphere.
\end{abstract}

Keywords: stars: abundances - stars: late-type - Galaxy: disc-Galaxy: evolution.

$\mathrm{Mo}$ and $\mathrm{Ru}$ as neutron-capture elements are produced by three different processes, namely the rapid and slow neutron capture process (respectively, the $\mathrm{r}$ and $\mathrm{s}$ processes) and the p-process. In this work we present and discuss the observations Mo and Ru abundances for FGK dwarfs, located in the Galactic disc with metallicity $-1<$ $[\mathrm{Fe} / \mathrm{H}]<+0.3$. The data obtained will allow exploring ways of production and enrichment by $\mathrm{Mo}$ and $\mathrm{Ru}$ of the galactic substructures and the Galaxy generally.

\section{Observations and atmospheric parameters}

The high-resolution echelle spectrographs SOPHIE ( $\mathrm{R}=$ $75000)$ and ELODIE $(\mathrm{R}=42000)$ on board $1.93 \mathrm{~m}$ telescope of OHP (France) for the wavelengths range 4400$6800 \AA \AA$ were used. Spectral processing carried out by (Katz et al., 1998; Galazutdinov, 1992).

The effective temperatures $\mathrm{T}_{\text {eff }}$, the surface gravities $\operatorname{logg}$, the microturbulent velocity $\mathrm{V}_{\mathrm{t}}$, and metallicities of the studied stars were determined earlier in our paper (Mishenina et al., 2013). Effective temperatures $\mathrm{T}_{\text {eff }}$ were estimated by the line depth ratio method (Kovtyukh et al., 2003). Surface gravities $\lg g$ were obtained by two methods: parallaxes and ionization balance of iron. a)

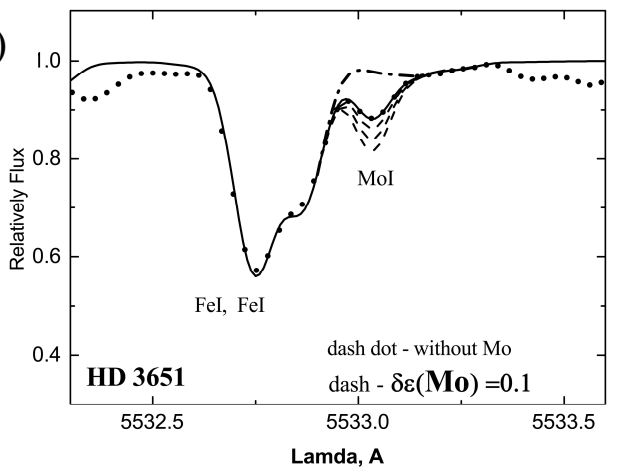

c)

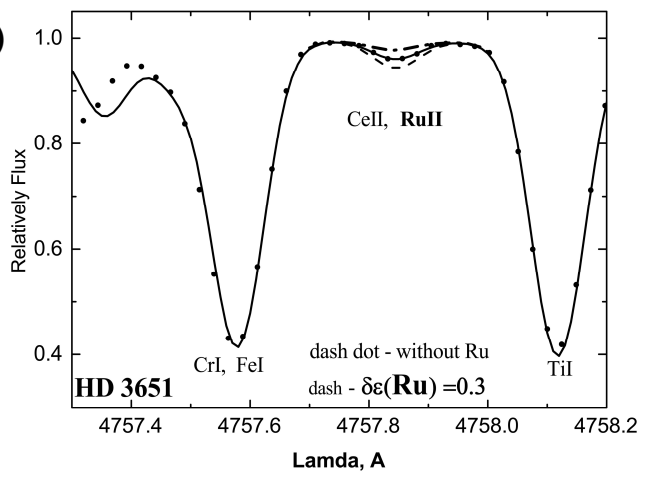

b)
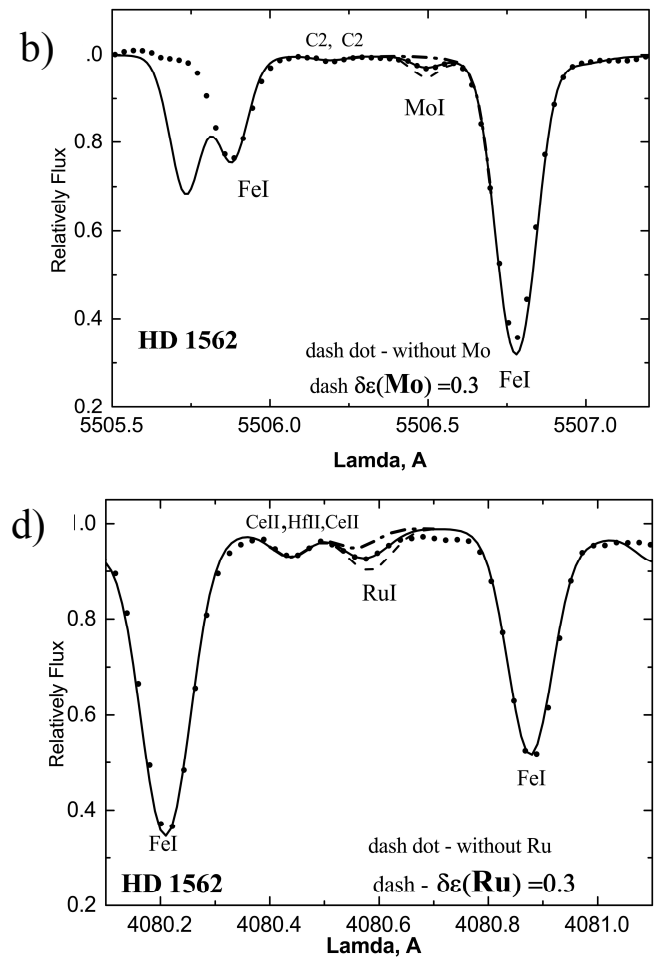

Figure 1: Comparison of synthetic and observed spectra in the region of MoI and RuI lines. Dotted line: observations; solid black lines marked the spectra calculated for the resulted abundances. 
a)

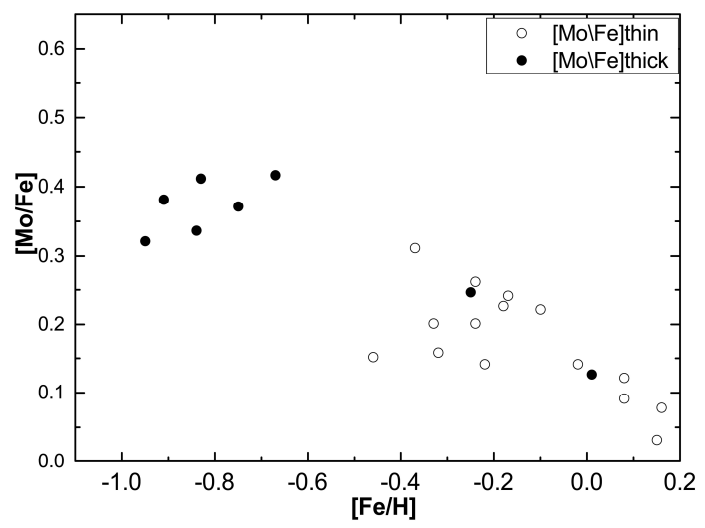

b)

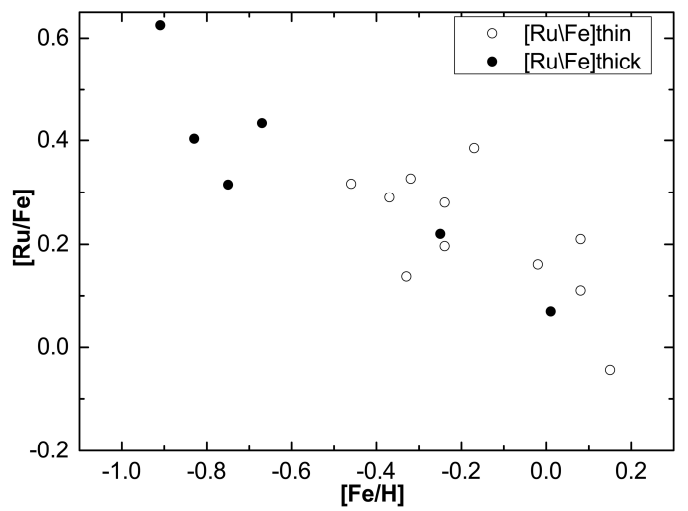

Figure 2: The run of $[\mathrm{Mo} / \mathrm{Fe}],[\mathrm{Ru} / \mathrm{Fe}]$ with $[\mathrm{Fe} / \mathrm{H}]$

\section{Abundances}

The following lines, detected in the spectra of the Sun, were considered for abundance determinations in star spectra: $\lambda 5506.493, \lambda 5533.031$ for MoI and $\lambda 4080.594$, $\lambda 4584.443$, and $\lambda 4757.848$ for RuI. Determination of the Mo and Ru abundances was made by STARSP LTE spectral synthesis code (Tsymbal, 1996). We used lines, which are weak and do not require to take into account the hyperfine or isotopic structure. The example of comparison of synthetic and observed spectra for MoI and $\mathrm{RuI}$ line is shown in Fig. 1.

\section{Results and conclusions}

Rapid (r) and slow (s) captures of neutrons to some extent describe the behavior of heavy elements. However, recent observations of the distribution of heavy elements in the oldest stars show the limits of this approach, in particularity, overabundance of Mo and Ru (Peterson, 2011). Some stars require processes with a neutron mean density between these processes, which are called the intermediate i process (Jones et al., 2016). Abundances and behavior of underinvestigated elements, such as molybdenum and ruthenium in stars of different metallicities and in various components of the Galaxy permits to study their contribution to enrichment in stars of thin and thick disks and to investigated the sources of their origin.
Here we investigated a small number of stars of galactic discs, and we can preliminary draw conclusions :

1) the abundances of the molybdenum and ruthenium decrease with increasing metallicity in both discs,

2) the abundance of these elements in the thick disc is higher than in the thin one.

In the future, we plan to make a determination of Mo and $\mathrm{Ru}$ abundances for a larger number of stars and also we will be performed an analysis from the point of view of the theories of nucleosynthesis and chemical evolution.

Acknowledgments. This work was supported by the Swiss National Science Foundation (SCOPES project No. IZ73Z0-52485).

\section{References}

Jones S., Ritter C., Herwig F. et al.: 2016, MNRAS, 455, 3848. Galazutdinov G.A.: 1992, Preprint SAO RAS, n92.

Katz D., Soubiran C., Cayrel R. et al.: 1998, $A \& A, 338,151$. Kovtyukh V., Soubiran C., Belik S. et al.: 2003, $A \& A$, 411, 559.

Mishenina T., Pignatari M., Korotin S. et al.: 2013, $A \& A$, 552, 128.

Peterson R.: 2011, ApJ, 742, id.21.

Tsymbal V.V.: 1996, ASP Conf. Ser., 108, 198. 